\title{
Supporting Narrative Understanding of Children with Autism: A Story Interface with Autonomous Autobiographic Agents
}

\author{
Wan Ching Ho, Megan Davis and Kerstin Dautenhahn
}

\begin{abstract}
Recent studies in education for children with autism demonstrated that computer assisted learning can increase children's narrative understanding. However, software interfaces which include autonomous intelligent agents have yet to be explored in this context. In this paper we investigate how a narrative storytelling environment which is populated with virtual characters may help children with autism to understand narrative structure and to remember meaningful events within in a story. The behaviours of characters in the narrative storytelling environment are driven by a cognitive agent architecture and, in particular, a specially designed computational autobiographic memory (AM) which allows the virtual characters to remember and express emotional experiences. An application named Virtual Agent Story Interface (VASI) has been developed to visualise the agents' AMs and to help the children to reconstruct their own version of a story. In this paper we describe related research in the area, the overall design rationale of VASI, details of the VASI software design and the preliminary evaluation study.
\end{abstract}

\section{INTRODUCTION}

As a novel tool for simulating human societies, multiagent systems [1] may help shed some light on various kinds of narrative and communication processes. Following this trend of research, we aim at simulating the reconstruction of personal life stories from past experiences remembered in personal autobiographic memory using a bottom-up and developmental perspective, and finding out how this simulation can assist children with autism in learning about narrative structure and meaningful events in stories. Humans are storytelling beings, by telling stories to ourselves and others we make sense of the social world [2]. However, people with autism have difficulties with narrative and consequently appear to live in a less coherent world [3], [4]. Nevertheless, a large body of research has shown that narrative is a good way of assessing linguistic ability in older children with communication impairments, including those with autistic spectrum disorders (see [5] and [6] for an overview). Moreover, recent studies have shown that computer assisted learning can increase the understanding of narrative demonstrated by children with autism [7], [8], [9]. However, there seems to be very little consideration of software interfaces with autonomous intelligent agents in this educational context. With our interest in modelling cognitive agents in computer software as an assistive technology for children with autism and other cognitive communication impairments, we aim to create characters with certain simplified aspects of human

Authors are with Adaptive Systems Research Group, School of Computer Science, University of Hertfordshire, Hatfield, Hertfordshire, AL10 9AB, UK.

\{w.c.ho, m.davis, k.dautenhahn\}aherts.ac.uk cognition, such as emotion and episodic memory, and enable them to act out some meaningful stories in an interactive narrative environment. To achieve this goal, we will need to answer the following three main research questions:

- How can intelligent agents help children with autism to understand narrative?

- What kind of memory/story structure do these agents need to possess?

- What design considerations need to be taken into account when developing this type of software (considering, for example, the information display, or the interactivity of the interface)?

The rest of this paper is organised as follows: For the background of our interdisciplinary research, we first introduce autism and its narrative impairments. We then review literature in the areas of the psychological concepts named autobiographic memory (e.g. [10], [11]), narrative psychology [12], and narrative intelligence [13]. We illustrate the considerable similarities among the main characteristics of research work in these areas and then discuss the importance of taking these similarities into account to enhance both the cognitive ability and believability of intelligent agents.

Following that we present a computational framework to model autobiographic memory (AM) for producing the narrative of agents' life stories in the context of humanagent interaction, taking into account how such a low-level approach may increase our understanding of the creation of personal narrative from our past significant experiences. Next we present the potential utilisation of an interactive narrative application, VASI (Virtual Agent Story Interface), with autobiographic agents to support the learning process in special user groups with narrative and communication disorders, such as children with autism.

\section{AUTISM AND ITS ASSISTIVE TECHNOLOGIES}

Autism is a lifelong developmental disability. There are three main areas of difficulty which all people with autism share - sometimes referred as the "triad of impairments" [14], [15], [16]:

- Impairment in communication

- Impairment in social interaction

- Impairment in imagination and fantasy

Narrative impairments have been shown in children with autism which include difficulties in producing a) narrative with a reasonable size and structure, for example often long, circular, and repetitious accounts of narrative events are created, and b) evaluation of the story, for example bizarre 
explanations of causality may be given and the emotions and mental states of characters not appreciated, that is they exhibit a difficulty with the so called Theory of Mind [17], [18], [19]. Autobiographic accounts, such as those by Temple Grandin, describe the impact that difficulties with narrative have on the everyday lives of people with autism [20], [21].

Investigation leading to a better interpretation of autistic children's narrative understanding has attracted many researchers from different areas such as Developmental Psychology and Linguistics. Many previous studies such as [5] and [6] used picture books to study children's narrative comprehension and writing skills.

The advancement of computer technology in recent years has helped researchers investigating educational or training environments to incorporate in their experimental tools the advantages conferred by a computer system, i.e. primarily predictability and controllability, but also time- and behaviour-logging can be easily enabled. Finally session management is simplified, and thus there are fewer burdens on the attending adults (the experimenters) and more attention can be given to the child participants.

Recent research has confirmed the usefulness of computerbased education in autism. Researchers developed and evaluated a computer-animated tutor to teach vocabulary and grammar which was used with autistic children [8]. It was also shown that computer training is useful to teach social skills to people with autism [7]. To take this one step further, researchers attempted to define a methodology to design educational software for autism - they tested the interfaces configured with various modalities: text, $2 \mathrm{D}$ or $3 \mathrm{D}$ images, synthetic voice, etc. [9]

In addition to the previous studies, it was found some children with autism can engage more in a collaborative narrative with an animated character, that is, with a virtual peer, than with a human peer [22]. Note that a "Wizard of Oz" (remote-control) methodology was used in the study to allow the experimenter to select responses from a range of options for the virtual peer during the collaborative narrative task.

Other research projects such as AURORA ${ }^{1}$ (AUtonomous RObotic platform as a Remedial tool for children with Autism) and IROMEC ${ }^{2}$ (Interactive RObotic social MEdiators as Companions) used focussed studies to investigate the potential enhancement of the everyday lives of children with autism (or with other disabilities) through the use of robots and other interactive systems as therapeutic or educational "toys".

Developed within the AURORA project, TouchStory is interactive software to help children with autism to understand narrative [23]. It presents primitive components of narrative as picture sequences on a touch screen and allows children to complete the sequences. The central idea of the interaction with this software is that narrative is fundamental to social comprehension and interaction - by

\footnotetext{
${ }^{1}$ http://www.aurora-project.com. Last accessed: 25-01-2009.

${ }^{2}$ http://www.iromec.org. Last accessed: 25-01-2009.
}

fitting events into a narrative pattern, users can construct and inhabit a meaningful social world [4], [24], [25], [12], [26]. Our research work in this paper follows the direction of TouchStory and introduces characters acting in the story with a cognitive model of "mind".

\section{AUTOBIOGRAPHIC AGENTS AND NARRATIVE CONSTRUCTION}

In everyday life humans automatically and instinctively apply interpretive strategies on perceiving stories from different types of media. These interpretive strategies which represent knowledge about particular situations or events can be derived directly from past experiences, including stories told by and heard from other individuals. These strategies depend not only on story stereotypes ${ }^{3}$ (schemas or prototypes of stories in our memory that associate a pattern of cues with a typical set of stories), but also on personal experiences in autobiographic memory (AM). The latter can be essential to the development of these strategies - thus creating powerful social biases that can have an impact on retelling the stories.

$\mathrm{AM}$ is a specific kind of episodic memory [10] and contains significant and meaningful personal experience for a human being. It can also be seen as mental constructions of the self and very often features imagery while simultaneously containing abstract personal knowledge [28], [29], [30].

As one of the primary functions of AM is to share personal experience with others through storytelling [10], in our previous work we created computational models of AM for both Artificial Life agents [31] and synthetic characters in interactive narrative environments [32]. The concept of Autobiographic agent was first defined in [33, page 5] as "an embodied agent which dynamically reconstructs its individual 'history' (autobiography) during its life-time."

In the sense of narrative construction, autobiographic agents are therefore different from other types of storytelling agents which are, if not remotely controlled, usually preprogrammed either with temporal and structured stories or by simultaneously selecting story sequences from a large story database. Agents with AM can also avoid the problem of behavioural incoherence as described by Sengers [34] - human observers are usually not able to understand why agents behave in a particular way because their decision making and particularly their behaviour generation are derived from the use of their past experiences (that are usually unknown to the user).

\footnotetext{
${ }^{3}$ Stereotypes of stories can help people make quick decisions during story comprehension or retelling. By matching with stereotypes already in memory, people do not need to remember or recall every detail of the story "from scratch" and thus can save time and effort when retelling a story. A similar idea of "gist" was suggested by Schank [27] who suggested that the process of creating the story also creates a memory structure that will contain the gist of the story for the rest of our lives. Note, we may only speculate at this point, but people with difficulties in reconstructing stories may have fewer prototypes of stories available in their memory. Take as an example an important element of stories, the characters: children with autism may have fewer stereotypes to judge a character's intentions and likely actions because they do tend not to perceive these features while watching a story unfold.
} 
Overall, in character-based interactive storytelling environments, we explored the integration of a computational autobiographic memory into an agent architecture that already enables autonomous agents to "experience emotions", and behave believably accordingly to a consistent personality [35]. We also took inspiration from human life stories and illustrated an implementation for agents' storytelling memory structure, which essentially incorporates human narrative discourse units and the reconstructive nature of human autobiographic memory [32]. Through the creation of agents' own life stories, we expect that this memory can help agents to 1) express their internal subjective sense of "self" and 2) show continuity of the "self".

A more detailed overview for the interrelationship between autobiographic agents and narrative in the area of Intelligent Virtual Agents can be found in [32].

\section{VIRTUAL AGENT STORY INTERFACE (VASI)}

To investigate the narrative understanding of children with autism, an anti-bullying software named FearNot! that had been developed within a project named eCIRCUS ${ }^{4}$ (Education through Characters with emotional-Intelligence and Role-playing Capabilities that Understand Social interaction) was adapted to generate contents from a character's AM to feed into our newly developed Virtual Agent Story Interface (VASI).

The eCIRCUS project developed a new approach in the use of Information and Communication Technology (ICT) to support social and emotional learning within personal and social education. This approach was achieved through virtual role-play with synthetic characters that establish credible and empathic relations with the learners.

FearNot! was designed for typically developing children and provides them with the opportunity to visit a virtual school environment complete with characters representing the most significant roles in bullying scenarios that are commonplace in real-life bullying incidents. Users interact in the environment by watching narrative episodes unfolding as the result of the action executed by autonomous characters (the top screenshot in Figure 1). After each episode, the user can interact with one of the characters (the bottom screenshot in Figure 1), for example to give suggestions on how to cope with the problem, and then observe the result of the given advice in a subsequent episode.

The types of episodes shown in FearNot! will be familiar to many children with autism, as the National Autistic Society in UK indicate that over 40 per cent of children with autism have been bullied at school ${ }^{5}$. Therefore we expect that the storylines in FearNot! will be believable to most of the potential users of VASI. At the same time bullying scenarios are usually dramatic and thus they are "worth remembering and telling" [24] particularly, for children with autism, when compared with stories which are less realistic such as folk stories or fairy tales. Since characters with

\footnotetext{
${ }^{4}$ http://www.e-circus.org/. Last accessed: 25-01-2009.

${ }^{5}$ http://www.nas.org.uk/nas/jsp/polopoly.jsp?d=824\&a=11873. Last accessed: 25-01-2009.
}

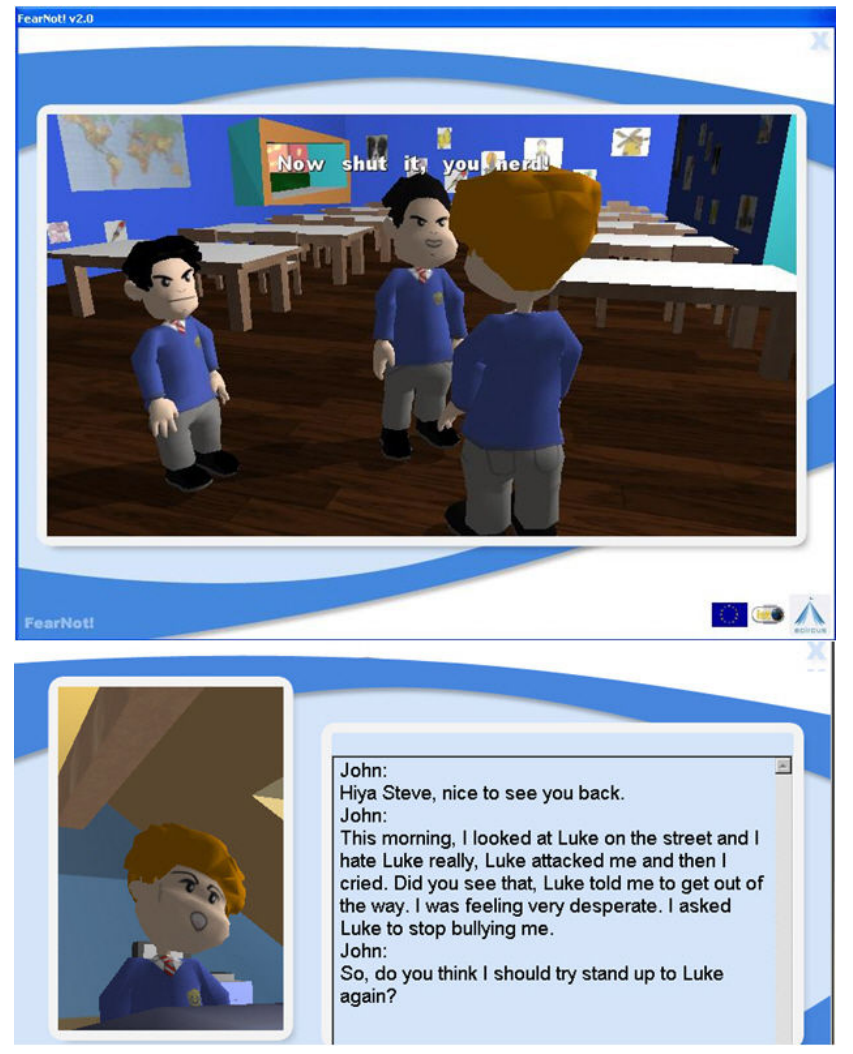

Fig. 1. Sample screenshots of FearNot! software: (top) a bullying episode and (bottom) user interaction interface.

their computational AM in FearNot! are able to remember meaningful events based on their current goals, actions and emotions, we can check at any point of the unfolding story what an individual character has remembered so far by transferring the memory (XML) file of this character to the VASI software. This process is shown in Figure 2, together with the interface of VASI.

\section{A. Technical Implementation for VASI}

VASI is newly developed software that serves as an narrative reconstruction tool though which children can easily find out what was going on inside a character's mind in the story which they have viewed. The wide compatibility provided by Java Virtual Machine means that VASI is largely platform-independent. Users can interact with VASI using mouse-clicks to select buttons with icons and a keyboard to type their own story. However, the user does not need to be familiar with typing, as described later. At the left-hand-side of the interface shown in Figure 2, different bullying episodes are displayed in the leftmost column and each episode can be expanded (by clicking on the episode picture) into a sequence of events displayed in the second column. Story episodes and events are displayed chronologically down both columns, so that children can have a clear idea about the order of occurrence.

VASI offers a text input area on the right-hand-side of the screen, in which the user can write the story using their own words and then click the Insert button to add the written text 


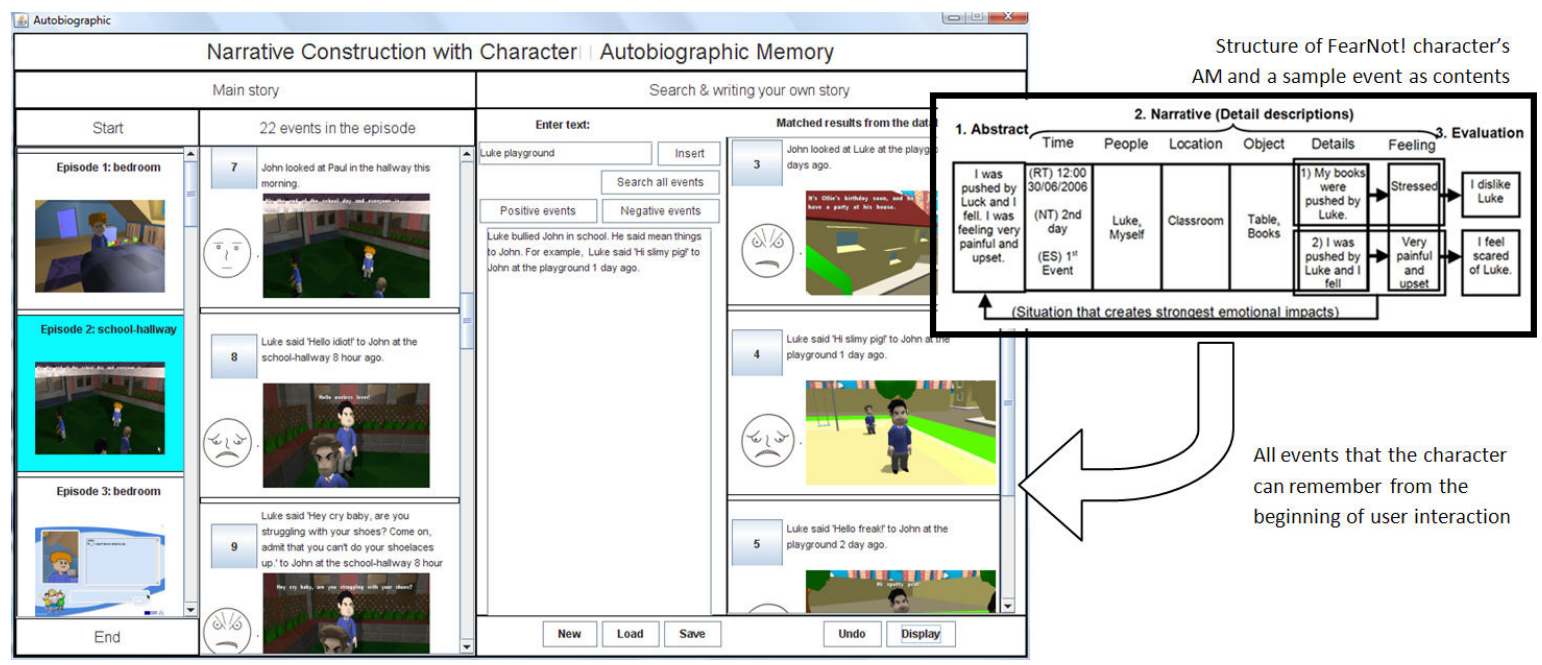

Fig. 2. Structure of character's AM (left), the interface of VASI (right), and the rough process of memory transfer for displaying experiences as events remembered by the character.

into the large story-display area. Alternatively, the user can type in any combination of keywords to perform a search throughout the character's AM for memorised events matching these keywords. In addition the buttons Positive events and Negative events allow the user to search emotionally positive or negative events for the character. The results of each search, if any, are displayed in the rightmost column and ranked based on the strength of the emotional impact of the particular event on the character - the stronger the emotional impact created by the event, the higher the position of that event in the display.

Each event is displayed with a sequence number (e.g. event No. 2 in the episode), which can be clicked to add the description of that event into the user's own story in the large story-display area. This feature allows VASI to be used by children who are not able to or who are not familiar with using a computer keyboard but still want to capture particular events into their own story. Thus their story can be built up piece by piece through clicking buttons representing individual events.

Agents in FearNot! have not only semantic knowledge of the world, which is provided directly from the perceptions of world properties, but they also remember episodic events kept in their AM. Figure 3 shows the event structure for an agent's AM. The design of this structure was inspired by the research on narrative structure in life stories for humans [2]. Each event remembered in an agent's AM has a level of significance to the agent, which is represented by the encoded emotional intensity associated with that event. The level of significance is mainly used for representing the priority of an event during the ranking process described previously. Event reconstruction takes place in the memory retrieval process. As a result, a context-dependent memory schema is generated when an agent tries to remember events in its memory in each situation (see Figure 4). Therefore, AM is used for generating summaries of "personal" history. In general, we predict that if agents can actively tell their own

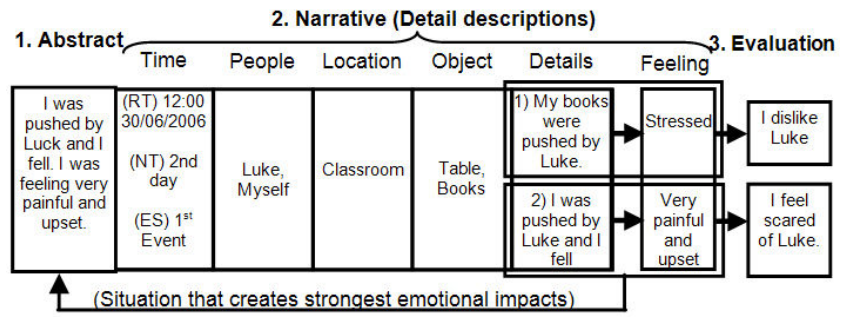

Fig. 3. Details of a sample event, constructed under an episode where an agent (Luke) hits another agent (John).

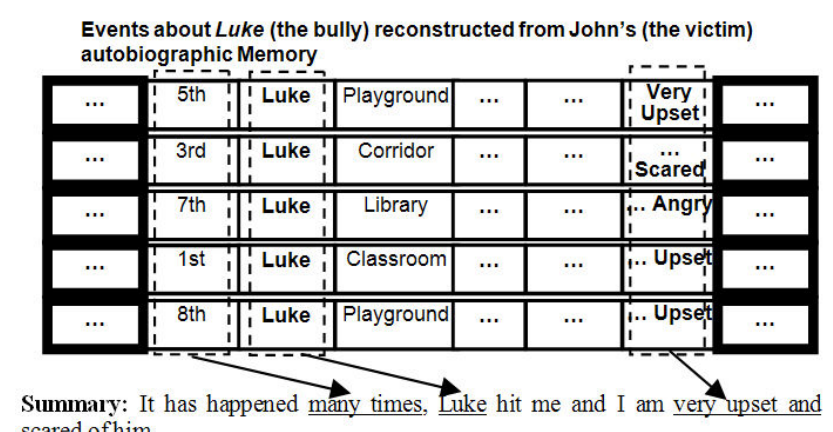
scared of him.

Fig. 4. How a summary of the agent past experiences can be generated.

summarised experiences as stories to the user, they will be more interactive and believable.

The description of each event is then generated from the language engine used in FearNot! to create the characters' conversation (see [36] for details). In order to produce descriptions with various lengths and structures, the language engine randomly omits one or more of the main elements of narrative (e.g. time, place, characters involved, what happened and the character's feelings; as shown in the upper part of Figure 3).

Finally, one of the simplified emotion icons adapted from the study in [37], is attached to each event to explicitly 
display the internal states which the character was experiencing at that specific time. This feature is important in allowing children with autism, who have a deficit in reading characters' minds, to understand how the character was feeling during the event. The displays of the emotion icons and event descriptions in VASI further distinguish our study from others which use only picture books or animated characters on a computer screen. First, animated characters cannot display emotions as richly as real humans do, and children with autism may not pay much attention to features on the characters' faces during the story comprehension task. Second, static picture books may not give rich enough context to show what a story character really feels, even when the character's facial expression is drawn on the page.

As found in an earlier study [38], in the absence of explicit direction, children with autism were less attentive to facial expressions of emotion than children from a control group. However, this difference disappeared when children were explicitly asked to make a socially relevant decision as more contextual information was given to them. With these findings researchers suggested that the attention of children with autism to the emotions expressioned by others is influenced by situational factors. Here VASI provides an easy and interactive software interface to maximise the situational factors in the story (e.g. chronological orders of events, relevant descriptions and character's emotional states) using a character's AM which contains its past experiences that are worth reporting to the user.

\section{B. Design Considerations}

We learned from the literatures, such as those discussed in earlier sections, about the general cognitive (e.g. perceptual) deficits of children with autism. While developing the VASI interface which allows the children to interact over a long period of time, we put together a list of design considerations, as follows:

- Providing better social engagements using autonomous virtual characters

- Reminding users of events from the story he or she has watched by using screen shots of FearNot!

- Allowing children to use VASI in a computer as it is a predictable and safe environment for free interactions

- Addressing the deficit of writing narrative, in particularly the evaluation part, within the narrative structure

- Designing characters that can tell their story from their own perspective, and actively tell the user about their emotions towards events and other characters

- Using an interface with black \& white icons showing characters' emotion to reduce the cognitive load for autistic children

- Designing a final display of the finished written story as a reward screen for users (see the bottom-right part of the screenshot of VASI in Figure 2)

Unlike designing an educational software or a simple computer game for typically developing children, these design considerations are critical to the design of VASI in order to address the needs of our user group. Some of them were

\begin{tabular}{|c|c|c|}
\hline Visit No. & Activity & Purpose \\
\hline Visit 1 & Narrative comprehension & Pre-trial measure \\
\hline Visit 2 & Video $1 \&$ VASI & Introductory week \\
\hline Visit 3 & Repeated video 1 \& VASI & Emphasise predictability \\
\hline Visit 4 & Video 2 \& VASI & $\begin{array}{l}\text { Increase content variety } \\
\& \text { maintain interest }\end{array}$ \\
\hline Visit 5 & Video 3 \& VASI & $\begin{array}{l}\text { Increase content variety } \\
\& \text { maintain interest }\end{array}$ \\
\hline Visit 6 & Video 4 \& VASI & $\begin{array}{c}\text { Increase content variety } \\
\& \text { maintain interest }\end{array}$ \\
\hline Visit 7 & Narrative comprehension & Post-trial measure \\
\hline
\end{tabular}

TABLE I

EXPERIMENTAL PROTOCOL FOR OUR PRELIMINARY VASI EVALUATION STUDY.

also applied (and thus validated) in our previous studies with autistic children [39] (see next section for more details).

\section{EVALUATION STUDY}

As described in the previous section, VASI was developed and evaluated along with FearNot!. While our main focus is on the development of an interactive system which addresses the needs of children with autism, we are also interested to study whether it will be effective for other children with difficulties in comprehending narrative. In this initial exploratory study, weekly evaluations were carried out over an extended period with six children aged between 4 years and 9 years from a after-school club ${ }^{6}$. Four of these children had diagnoses of autism. The other two were diagnosed with behaviours suggestive of autism and one of them was alsodiagnosed with attention-deficit/hyperactivity disorder(ADHD) and speech and language difficulties ${ }^{7}$. Our participants were not regarded as a representative experimental sample, rather they were seen as individual cases.

Table I illustrates the experimental protocol for our preliminary evaluation study. Visits 1 and 7 (which were in fact 10 weeks apart due to holidays etc.) were designated to pre- and post-trial narrative comprehension measures, in which each participant spent the first 3 minutes watching a video and then filled in a on-screen questionnaire. Results we collected from both measures will be analysed with the narrative comprehension criteria ${ }^{8}$ from Paris and Paris [40]. From Visit 2 to 6 , each participant first spent around 5 minutes watching a FearNot! video clip and observed how bullying stories unfold with virtual characters in a virtual environment. They then spent another 10 to 15 minutes writing their own story using VASI. At any time of any session, participants were free to quit the session or the whole study if they wanted to without providing any justifications.

\footnotetext{
${ }^{6}$ The after-school club, which is for children with autism and their families, draws from a number of schools in a wide area. In line with the ethos of the AURORA project we took an open inclusive policy to participants - no participant who was offered by the after-school club was rejected for the study.

${ }^{7}$ The after-school club provided this information - we did not have access to more detailed diagnostic data.

${ }^{8}$ The reason we used the comprehension criteria from Paris and Paris [40] is that, compared with other sources, they have shown that different "actual stories" may be used.
} 
Note that FearNot! video 1 was used in both visit 2 and visit 3 to enhance predictability allowing participants to get used to the FearNot! stories as well as VASI at an early stage of the evaluation study. From visit 4 onwards we gradually changed the content of the videos to maintain participants' interest and enable them to remember a larger variety of episodes during their story re-writing exercise with VASI.

Since VASI allows the children to see the contents of a character's memory, that is the character's goals, actions and feelings at specific moments in the stories previously seen in FearNot!, we hope that VASI can increase the children's understanding of the character's internal states (e.g. emotions, goals, intentions) and so enhance understanding of others. Our expectations are that VASI can help children with autism to learn how to create better narratives, in the sense of "meaningful and reportable narratives" that are important for social interaction and communication with others.

While the data analysis is still on-going, our early observations suggest that participants' interest levels were sustained; during the evaluation period they tried to attend every session. At later stages of the evaluation, some children actively talked to the experimenter at the beginning of a session about characters and episodes from their previous interactions with VASI. However, due to the preliminary nature of this evaluation study, it is too early to comment on any effect of VASI on participants' day to day experience.

If children in our evaluation can reconstruct the FearNot! stories with some specific content that is unique to the individual child, then this may indicate that the interface has succeeded in allowing children with autism to perceive a story "personally". Towards this goal, we plan to assess the written narrative contents and structures from these children at the beginning and the end of the evaluation. We will focus on the analysis in particular the global narrative (story) structure and children's ability to provide evaluative comments, e.g. mental or emotional states of the story characters. It will be interesting to investigate participants' understanding of the "ingredients of story" such as, causality, irreversibility, temporal sequence, character continuity etc. In previous work one author of this paper introduced t-stories, which are simple game-like tasks which address the primitive components of narrative (that is, story ingredients) in a human-computer interaction context, using a purpose designed software game called TouchStory [39]. The underlying conceptual framework being to investigate to what extent the participants' understanding of narrative could be improved by allowing them to focus on the primitive components of narrative, in contrast to fully elaborated stories. It was found that some participants did learn from TouchStory, in that they became more able to complete previously unseen t-stories (that is they demonstrated a better understanding of the components of narrative). The analysis of the current work intends to build on this basis.

\section{CONCLUSIONS AND FUTURE WORK}

In this paper, we proposed an interactive narrative storytelling interface called VASI which uses a computational autobiographic memory designed for intelligent virtual characters, and discussed how it can assist children with autism in understanding narrative structure and thus remembering meaningful events in stories. Our work is grounded in the importance of personal life stories in creating a coherent world view, and difficulties encountered when there is a deficit in narrative ability, such as in the case of autism [3], [19]. Based on our previous research into Artificial Life agents, believable characters in interactive narrative systems, and interactive software designed to help children with autism to understand narrative; we discussed how computational autobiographic memory can facilitate intelligent agents in storytelling as well as communicating with human users.

In the near future we will be able to carry out data analysis for the results we collected from our long-term weekly study with individual users. We hope that the outcome of this task can give us a deeper insight into the deficits of narrative understanding in children with autism and enable us to develop interactive software which is better suited to facilitate their narrative understanding and help with particular individual difficulties.

Since a computational "mind" equipped with better story understanding may help children to improve their communication and social skills [12], [17], our future research will also focus on the enhancement of an interactive virtual environment ${ }^{9}$, in which users will be allowed to interact and communicate with a group of social agents in a school environment for enabling the social relationship to be emerged.

\section{ACKNOWLEDGMENTS}

This work was partially supported by European Community (EC) and is currently funded by the eCIRCUS project IST-4-027656-STP with university partners HeriotWatt, Hertfordshire, Sunderland, Warwick, Bamberg, Augsburg, Wuerzburg plus INESC-ID and Interagens. The authors are solely responsible for the content of this publication. It does not represent the opinion of the EC, and the EC is not responsible for any use that might be made of data appearing therein.

\section{REFERENCES}

[1] M. Wooldridge, An Introduction to MultiAgent Systems. John Wiley \& Sons Ltd, 2002.

[2] C. Linde, Life Stories: The Creation of Coherence. Oxford University Press, 1993

[3] J. Bruner and C. Feldman, "Theories of mind and the problem of autism," in Understanding other minds: perspectives from autism, S. Baron-Cohen, Ed. Oxford University Press: Oxford, 1993.

[4] K. Dautenhahn, "The origins of narrative - in search for the transactional format of narratives in humans and other social animals," ognition and Technology: Co-existence, Convergence, Co-evolution (IJCT), pp. 97-123, 2002, john Benjamins Publishing Company.

[5] C. Norbury and D. Bishop, "Narrative skills in children with communication impairments," International Journal of Language and Communication Impairments, vol. 38, pp. 287-313, 2003.

[6] L. Stirling and G. Barrington, "'then i'll huff and i'll puff or i'll go on the roff" thinks the wolf: Spontaneous narratives written by a child with autism," in Mental states: Language and cognitive structure., A. Schalley and D. Khlentzos, Eds. Amsterdam/Philadelphia: John Benjamins, 2008, pp. 133-171.

\footnotetext{
${ }^{9}$ This future research is based on our previous work in [41].
} 
[7] V. Bernard-Opitz, N. Sriram, and S. Nakhoda-Sapuan, "Enhancing social problem solving in children with autism and normal children through computer-assisted instruction," Jounal of Autism and Developmental Disorders, vol. 31, no. 4, pp. 377-384, 2001.

[8] A. Bosseler and D. W. Massaro, "Development and evaluation of a computer-animated tutor for vocabulary and language learning in children with autism," Journal of Autism and Developmental Disorders, vol. 33, no. 6, p. 653V672, 2003.

[9] O. Grynszpan, J. Martin, and J. Nadel, "Designing educational software dedicated to people with autism,' in Assistive Technology: From Virtuality to Reality (Proceedings of AAATE 2005), A. Pruski and H. Knops, Eds. Lille, France: IOS Press, 2005, pp. 456-460.

[10] K. Nelson, "The psychological and social origins of autobiographical memory," Psychological Science, vol. 4, pp. 7-14, 1993.

[11] M. A. Conway, "A structural model of autobiographical memory," in Theoretical Perspectives on Autobiographical Memory, M. A. Conway, D. C. Rubin, H. Sinner, and E. W. A. Wagner, Eds. Dordrecht, the Netherlands: Kluwer, 1992, pp. 167-194.

[12] J. Bruner, "The narrative construction of reality," Critical Inquiry, vol. 18, no. 1, pp. 1-21, 1991.

[13] M. Mateas and P. Sengers, Eds., Narrative Intelligence, ser. Advances in Consciousness. Amsterdam: John Benjamins Publishing Company, 2002.

[14] U. Frith, Explaining the enigma. Blackwells, 1989.

[15] S. Powell, "Autism," in Developmental psychology, D. Messer and S. Millar, Eds. Cambridge: Cambridge, 1999, pp. 243-261.

[16] L. Wing, The autistic spectrum: A guide for parents and professionals. London: Constable, 1996.

[17] S. Baron-Cohen, Mindblindness. Cambridge, MA: The MIT Press., 1994.

[18] F. Abell, F. Happé, and U. Frith, "Do triangles play tricks? attribution of mental states to animated shapes in normal and abnormal development," Cognitive Development, pp. 1-15, 2000.

[19] L. Capps, M. Losh, and C. Thurber, "The frog ate the bug and made his mouth sad; narrative competence in children with autism," Journal of Abnormal Child Psychology, vol. 18, pp. 193-204, 2000.

[20] T. Grandin, Thinking in Pictures. New York: Doubleday, 1995.

[21] O. Sacks, An Anthropologist on Mars. Picador, 1996.

[22] A. Tartaro and J. Cassell, "Playing with virtual peers: Bootstrapping contingent discourse in children with autism," in Proceedings of International Conference of the Learning Sciences (ICLS). Utrecht, Netherlands, June 24-28 2008.

[23] M. Davis, K. Dautenhahn, C. L. Nehaniv, and S. D. Powell, "The narrative construction of our (social) world: steps towards an interactive learning environment for children with autism," Journal of Universal Access in the Information Society, vol. 6, no. 2, pp. 145 - 157, 2007.

[24] J. Bruner, Actual Minds, Possible Worlds. Harvard University Press, 1986.

[25] — Acts of meaning. Harvard University Press, 1990.

[26] R. Schank, "Tell me a story," in Narrative and intelligence, M. Mateas and P. Sengers, Eds. Northwestern University Press, 1990.

[28] M. A. Conway, Autobiographical Memory: An Introduction. Buckingham: Open Univ. Press, 1990.

[29] - "Autobiographical memories and autobiographical knowledge," in Remembering our past: Studies in autobiographical memory, D. C. Rubin, Ed. Cambridge, UK: Cambridge Univ. Press, 1996, pp. 67-93.

[30] M. A. Conway and C. W. Pleydell-Pearce, "The construction of autobiographical memories in the self memory system," Psychological Review, vol. 107, pp. 261-288, 2000.

[31] W. Ho, K. Dautenhahn, and C. L. Nehaniv, "Computational memory architectures for autobiographic agents interacting in a complex virtual environment: a working model," Connection Science, vol. 20, no. 1, pp. $21-65,2008$

[32] W. Ho and K. Dautenhahn, "Towards a narrative mind: Creating coherent life stories for believable agents," in Intelligent Virtual Agent (IVA) 2008, 2008, pp. $59-72$.

[33] K. Dautenhahn, "Embodiment in animals and artifacts," in AAAI FS Embodied Cognition and Action. AAAI Press, 1996, pp. 27-32, technical report FS-96-02.

[34] P. Sengers, "Narrative and schizophrenia in artificial agents," in Narrative Intelligence, M. Mateas and P. Sengers, Eds. Amsterdam: John Benjamins, 2003, pp. 259-278.

[35] W. C. Ho, S. Watson, and K. Dautenhahn, "Amia: A knowledge representation model for computational autobiographic agents," in
Proceedings of IEEE International Conference on Development and Learning (ICDL), 2007.

[36] J. Dias, W. Ho, T. Vogt, N. Beeckman, A. Paiva, and E. Andre, "I know what i did last summer: Autobiographic memory in synthetic characters," in Affective Computing and Intelligent Interaction, vol. Volume 4738/2007 of Lecture Notes in Computer Science. Springer Berlin/Heidelberg, 2007, p. 606V617.

[37] P. Howlin, S. Baron-Cohen, and J. Hadwin, Teaching Children with Autism to Mind-Read: A Practical Guide for Teachers and Parents. John Wiley and Sons, 1998.

[38] S. Begeer, C. Rieffe, M. Terwogt, and L. Stockman, "Attention to facial emotion expressions in children with autism," Autism, vol. 10, no. 1, pp. 37-51, 2006.

[39] M. Davis, "Touchstory: Interactive software designed to assist children with autism to understand narrative," Ph.D. dissertation, School of Computer Science, Faculty of Engineering and Information Sciences, University of Hertfordshire, 2009.

[40] A. Paris and S. Paris, "Assessing narrative comprehension in young children,” Reading Research Quarterly, vol. 38, no. 1, pp. 36-77, 2003.

[41] S. Watson, K. Dautenhahn, W. C. Ho, and R. Dawidowicz, "Developing relationship between autonomous agents: promoting pro-social behaviour through virtual learning environment," in Handbook of Research on Agent-Based Societies: Social and Cultural Interactions, G. P. Trajkovski and S. G. Collins, Eds. IGI Global, 2009. 